\title{
A Neuroscience Agenda for Counseling Psychology Research
}

\author{
Óscar F. Gonçalves \\ Northeastern University and University of Minho
}

\author{
Kristin M. Perrone-McGovern \\ Ball State University
}

\begin{abstract}
Recent advances in the field of neuroscience have dramatically changed our understanding of brainbehavior relationships. In this article, we illustrate how neuroscience can provide a conceptual and methodological framework to understand our clients within a transdiagnostic developmental perspective. We provide directions for integrating neuroscience into future process and outcome research. We present examples on how neuroscience can be integrated into researching the effects of contextual counseling interventions. We posit that interpersonal and environmental factors, such as neurotoxic factors (e.g., emotional neglect, stress), positive neurodevelopmental factors (e.g., nurturing and caring, environmental enrichment), and therapeutic interventions influence psychological processes (executive control, behavioral flexibility, reinforcement learning and approach motivation, emotional expression and regulation, self-representation and theory of mind). These psychological processes influence brain networks (attention, motivational, emotional regulation, social cognition), which influence cognitive, social, emotional, identity, and vocational development.
\end{abstract}

Keywords: counseling psychology, neuroscience, professional issues, brain-behavior relationships

The last decades have witnessed an impressive evolution of our understanding of the neurobiological basis of psychological processes. The discovery of neuroplasticity mechanisms was instrumental for understanding the influence of psychosocial factors on the brain. The pioneering work of Nobel Laureate Eric Kandel (2000) revealed that brain cells form new neural connections in response to novel learning experiences or exposure to new environmental stimuli. This phenomenon is referred to as neuroplasticity. Kandel (1998), himself, laid the ground for the clinical implications of the research on neuroplasticity by stating that:

Insofar as psychotherapy or counseling is effective and produces long-term changes in behavior, it presumably does so through learning, by producing changes in gene expression that alter the strength of synaptic connections and structural changes that alter the anatomical pattern of interconnections between nerve cells of the brain. As the resolution of brain imaging increases, it should eventually permit quantitative evaluation of the outcome of psychotherapy. (p. 460)

In fact, as the sophistication of neuroscience methodologies increased, we started to have consistent evidence that counseling and psychotherapy could alter the brain. One of the first reviews on the studies of the effect of psychotherapy on the brain was that of Kumari (2006), who concluded that:

Óscar F. Gonçalves, Department of Counseling and Applied Educational Psychology, Northeastern University, and School of Psychology, University of Minho; Kristin M. Perrone-McGovern, Department of Counseling Psychology and Guidance Services, Ball State University.

Correspondence concerning this article should be addressed to Óscar F. Gonçalves, Department of Counseling and Applied Educational Psychology, Bouvé College of Health Sciences, Northeastern University, 404 International Village, Boston, MA 02115, or Kristin M. PerroneMcGovern, Department of Counseling Psychology and Guidance Services, Teachers College, 6th Floor, Ball State University, Muncie, IN 47306. E-mail: o.goncalves@neu.edu or kperrone@bsu.edu
The data available so far, for example in depression, panic disorder, phobia and OCD, clearly suggest that a change in patients' symptoms and maladaptive behavior at the mind level with psychological techniques could potentially change (normalize) the brain at the functional level in the same way as faulty brain signals resulting from dysfunctional neural circuitries lead to psychopathological behavior. (p. 67)

A more recent review by Barsaglini, Sartori, Benetti, PetterssonYeo, and Mechelli (in press) went a step further by concluding that brain networks found to be dysfunctional in psychological disorders were normalized after effective psychotherapeutic intervention. Additionally, psychotherapy seems also to recruit compensatory networks (i.e., brain compensates for a deficit in one area by mobilizing alternative complementary brain regions). For example, Penadés et al. (2013) found that cognitive remediation therapy for persons with schizophrenia not only improves functioning of the brain networks affected in this disorder but also facilitates interhemispheric communication by increasing the connectivity in the anterior regions of the corpus callosum.

Finally, there seems to be some specificity of the psychotherapeutic interventions, which do not overlap completely with psychopharmacology interventions. For example, cognitive therapy reduces depression by increasing activation of the prefrontal regions and by this mechanism decreasing the activity of the limbic region. In contrast, antidepressant medication reduces depression by directly inhibiting limbic activity and, by this process, indirectly increases the activation of the prefrontal cortex (Goldapple et al., 2004).

Neuroimaging technologies (Sampaio, Soares, Coutinho, Sousa, \& Gonçalves, in press), along with other psychophysiological (Oliveira-Silva \& Gonçalves, 2011), neurophysiological (Pinheiro et al., 2013), and neuromodulation techniques (Leite, Carvalho, Fregni, Boggio, \& Gonçalves, 2013) have been used to refine our psychologically informed theories (Cacioppo, Berntson, \& Nusbaum, 2008). Thus, a deeper understanding of the biological mech- 
anisms underlying psychological processes has been gained through methodological advances, giving birth to a new discipline most often referred to as cognitive or affective neuroscience (Cowan, Harter, \& Kandel, 2000). More recently, counseling psychologists have asserted the need to integrate neuroscience contributions in counseling psychology research and practice (Ivey \& Zalaquett, 2011; Ivey, Ivey, \& Zalaquett, 2013). We agree with this assertion and further argue that counseling psychologists must play an active role in shaping the direction of future research in the area. Existing neuroscience research addresses the connection between emotions and neurocognitive processes, but we need to take this a step further to investigate the application and effectiveness of specific psychotherapeutic and preventative interventions.

As Buboltz, Deemer, and Hoffman (2010) noted, the content of the Journal of Counseling Psychology (JCP) as the preeminent scholarly journal in our field reflects the focus and values of counseling psychology as a discipline. Buboltz and colleagues reviewed the contents of JCP between 1999 and 2009 and found that the categories used in 1999 remained consistent for their 2009 analysis with the addition of one category: interpersonal relations, social support, and/or attachment. We foresee the addition of another category in 2019: that of applied affective neuroscience. Buboltz and colleagues also noted the important role of JCP in highlighting emerging shifts in scholarly emphasis needed to meet demands of changing global trends over the years. One such global trend that requires a shift in scholarly emphasis for counseling psychologists is the emerging understanding of brain-behavior relationships based on neuroscience research. In this article, we review recent neuroscience research relevant to counseling psychology and suggest specific directions for future research in applied affective neuroscience.

We argue that counseling psychologists should be leading this area of research, and we acknowledge that we have not as yet been as active as we could be in this area. We conducted a search of JCP using the PscINFO database and found zero results for the search terms neuroscience, fMRI, EEG, ERP, or brain; and only one article for the term neuropsychology. When we extended the search to include physiology, we found 13 articles that had been published in this area. We highlight one of these articles (Rochman \& Diamond, 2008) as a notable example of research that has the potential to inspire future counseling psychology research.

Rochman and Diamond (2008) attempted to identify physiological correlates (i.e., heart rate, finger temperature, and skin conductance) of unresolved anger and sadness. They used a task based on an emotion-focused experiential psychotherapy framework to elicit the emotions in college student participants who reported unresolved anger toward an attachment figure. The noted goal of this type of task was to help clients access more vulnerable feelings such as sadness that may be underlying their expressed anger. The authors are to be commended on this innovative research design in which physiological measures are used as a way to assess emotional functioning within the context of a specific therapeutic task. This approach extended past research on therapy process that used client self-report or observer ratings as well as past physiological research that assessed general emotional arousal but did not assess shifts between different emotions. Rochman and Diamond found increases in sympathetic arousal during the shift from anger to sadness but not with a shift from sadness to anger and discussed how this finding supported the construct of unfinished business (i.e., that unresolved anger is a marker of primary vulnerable emotions such as sadness that were avoided or not able to be experienced fully). Despite the limitations of this study (e.g., relatively small sample size; use of college student volunteers; laboratory environment), counseling psychology researchers could build on this research by examining, for example, the shift from anger to sadness during emotion-focused therapy in actual counseling sessions. Rochman and Diamond examined individuals who expressed their feelings toward an attachment figure who was not present, but we would hypothesize that the intensity of the emotions experienced by participants in the here and now of the therapy session would be higher when the attachment figure is present during the emotional expression. Future research could also be conducted in this area using neuroimaging and neurophysiology methods. For example, functional magnetic resonance imaging could be used to examine what regions in the brain are activated by specific emotion-focused therapy techniques, or event-related potentials could be used to measure distinct brain waves in relation to a specific change event in therapy. This would provide greater insight into the mechanisms by which psychotherapy interventions influence neuroplasticity.

In what follows, we provide some pathways for the integration of neurosciences in our counseling psychology research programs (see Figure 1). First, we illustrate how neuroscience can provide a conceptual and methodological framework to understand our clients within a transdiagnostic and developmental perspective that has been more akin to the counseling psychology field. Second, examples and suggestions for integrating neuroscience into future process and outcome research are provided. Third, we present
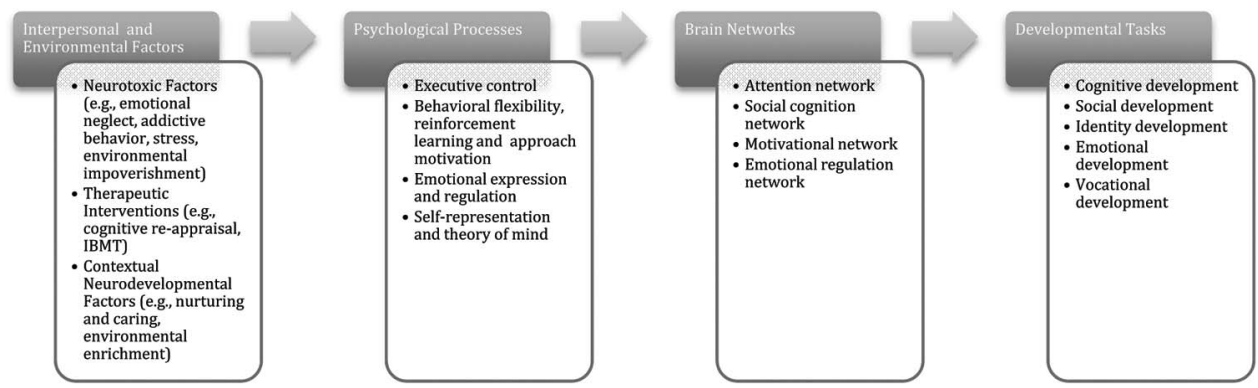

Figure 1. Pathways for the integration of neurosciences in counseling psychology research programs. IBMT $=$ integrative body-mind training. 
some examples on how neuroscience can be integrated into researching the effects of more contextual counseling interventions.

\section{Neurosciences Contributions for a Transdiagnostic and Developmental Approach}

Contrary to what happens in most neurological conditions in which local brain regions may underlie client symptoms, in psychological disorders, most symptoms are associated with highlevel integration and connectivity among different brain regions. As it was recently suggested by Buckholtz and Meyer-Lindenberg (2012), it is the alteration at the circuitry connectivity level where we can witness most of the cognitive, affective, and behavioral changes present in clients diagnosed with different psychological disorders. Building on current neuroscience research, these authors introduced a transdiagnostic model for psychopathology based on the idea of an altered brain connectivity in a configuration of four symptom-specific brain networks: attention and control network; reward and motivation network; affective arousal and regulation network; default mode social cognition network.

The attention control network is a frontoparietal network (i.e., connection between lateral prefrontal cortex, dorsal anterior cingulate, and dorsal parietal regions) involved mostly in executive functioning (e.g., working memory; goal-directed attention; performance monitoring). Common symptoms of deregulation of this network are inattention, working memory problems, and behavior programing deficits (e.g., schizophrenia, attention-deficit/hyperactivity disorder, major depressive disorder).

The reward and motivation network is a frontal striatal network (i.e., connection between lateral, orbitofrontal, and ventromedial prefrontal cortex and the subcortical regions of the striatum) found to be involved in several psychological processes such as behavioral flexibility, reinforcement learning, approach motivation, and salience attribution. Disruptions in this network result in symptoms such as impulsivity, compulsivity, and risk taking (e.g., obsessivecompulsive disorder; depression; personality disorders).

The affective arousal and regulation network is a corticolimbic network (i.e., connects lateral, orbitofrontal, ventromedial prefrontal cortex, and amygdala) underlying processes such as threat detection, affective responsiveness, emotional regulation, and fear extinction. Symptomatic expressions of deregulations of this system include anxiety, rumination, and emotional instability (e.g., mood disorders; anxiety disorders).

Finally, the social cognition network is a default mode network connecting the mechanisms involved in spontaneous rest activity between the temporal parietal junction, posterior cingulate cortex, and ventral medial prefrontal cortex, areas of the brain that are associated with self-representation and theory-of-mind processes. Disruption of these networks has symptomatic expressions such as social inadequacy, attachment deficits, or lack of empathy (e.g., autistic spectrum disorders).

According to the perspective discussed above, optimal developmental can be seen as the promotion of the brain networks associated with the four brain network systems: attention network, motivational network, emotional regulation network, and social cognition network. As exemplified in Figure 1, for counseling psychologists, it would be important to gain a better understanding, for example, of how the development of these four distinct networks are associated with the social emotional, cognitive, iden- tity, and vocational development across life span. Additionally, research is needed to investigate the potential neurotoxic impact on the different brain network systems of psychosocial factors such as emotional neglect, addictive behavior, stress, and environmental impoverishment.

For example, Tottenham et al. (2010) found that children residing in an orphanage for long time periods showed evidence of an increased amygdala volume associated with difficulties in emotional regulation, suggesting an alteration of the emotional regulation network. Several studies have shown that addictions, such as Internet addiction, sexual addiction, and gambling addiction, have been found to significantly alter cortical-subcortical connectivity in the motivational network (e.g., Hong et al., 2013; Katehakis, 2009; Sescousse \& den Ouden, 2013). Among other authors, Leskin et al. (2007) demonstrated that posttraumatic stress significantly impacted the attentional network executive indexes. Absent, however, from most of these studies is the developmental orientation needed to fully understand these network systems and their relationship to the normative developmental tasks that counseling psychologists are called to promote in their professional activities.

\section{Integrating Neuroscience in Counseling Process Research}

As aptly remarked by Kazdin (2007), "we know well that therapy 'works,' i.e., is responsible for change, but have little knowledge of why or how it works" (p. 2). The situation is not different from the one witnessed in the history of psychopharmacology. The first psychopharmacological treatments were empirically demonstrated long before we had a clear understanding of the active mechanisms present in each molecule (Valenstein, 1998). The difference between understanding that an intervention works and knowing the effective mechanisms responsible for its efficacy is the difference between evidence-based and science-based practice.

Counseling psychologists have a long tradition of examining the mechanisms responsible for change in the counseling process (Hill et al., 1988). The advent of neuroscience methods that allow for a moment-by-moment examination of discrete interventions may help counseling psychologists build on previous counseling process research. One example of an area that could advance traditional counseling process analogue research through the use of neuroscience is the study of mechanisms of emotional regulation (i.e., examining how we can help our clients switch between different emotional valences and arousal states). There is now growing evidence that the switching between emotional states is associated with the ability to activate different brain networks.

As it was recently pointed out by Tang et al. (2012), this ability to switch between different brain states is essential in helping self-regulate our cognitive and emotional interactions. Two emotional regulation mechanisms have been shown to impact brain functioning: cognitive reappraisal and mindfulness. Cognitive reappraisal consists of a process of reinterpreting an emotional trigger in order to produce an affective change (e.g., positive reframing). This is mostly a top-down regulation process that involves recruiting extended brain networks (e.g., frontal, parietal, and amygdala regions; McRae et al., 2012). This is a typical way by which counselors work with their clients, helping them reinter- 
pret the meaning of specific events. Most traditionally, cognitive reappraisal has been seen as a way of inducing, by a process of frontal executive control, a modulation of the amygdala response (regions that are part of the corticolimbic network discussed before).

However, a recent meta-analysis of the neuroimaging studies by Buhle et al. (in press) on the effects of cognitive reappraisal suggested a different picture. In fact, there is now abundant evidence showing the prefrontal control regions modulate the amygdala response by exerting control over the temporal cortical areas involved with the semantic representations of the experience. The recognition that the modulation of the amygdala response is dependent on the effective modulation of semantic systems has profound implications for developing more effective reappraisal and interpretation interventions. The results suggested reappraisal affects emotional regulation not by means of an executive shut down of the emotional experience but by a modulation of clients' meaning system.

An immediate implication for counseling psychology research would be to assess the ability of psychotherapists to effectively promote reconstruction of semantic and perceptual representations in the temporal cortical networks associated with emotional events. Specifically, counselors could build on the taxonomy of reappraisal tactics developed by McRae et al. (2012) to test the effects on brain regulation networks of prescribing (a) explicit positive reappraisal (e.g., what doesn't kill you makes you stronger), (b) a change of future consequences reappraisal (e.g., in a few years, I'll be laughing about this), (c) a distancing reappraisal (e.g., I don't have anything to do with it), or (d) an accepting reappraisal (e.g., these things happen, there was nothing I could do about it). These techniques could be compared in terms of desired therapeutic outcomes (e.g., decreased anxiety or depressive symptoms).

Additionally, future research could test (a) the differential effectiveness of self- (i.e., client) versus other- (i.e. therapist) generated interpretation in the brain networks associated with emotional regulation and (b) the effects of matching/unmatching of counselor's interpretation with the client's cultural representations in brain networks associated with emotional regulation.

Particularly important also is the finding that the way emotional regulation networks are impacted by cognitive reappraisal varies across development (Silvers, Buhle, \& Ochsner, 2013). For example, McRae et al. (2012) found that, as we reach adulthood, the process of cognitive reappraisal recruits an increased activation of the ventrolateral prefrontal cortex regions. Other brain regions, such as the medial prefrontal, posterior cingulate, and anterior temporal cortices, seem to follow a nonlinear process of development, with adolescents showing a pattern characterized by reduced activation during instances of unregulated emotions, in contrast to increased activation in the cognitive-reappraising process. This shows that the effectiveness of cognitive reappraisal processes is associated with the differential mobilizations of various regions of the emotional regulation system and may also differ across developmental tasks and domains. Future researchers could compare the effectiveness of cognitive reappraisal interventions across different developmental stages within domains such as identity development, social development, and vocational development.

One area in which counseling psychologists could examine this phenomena of variance in effectiveness of techniques such as cognitive reappraisal across different developmental stages is in the area of vocational development. For example, Super's (1980) theory of career development delineated stages of vocational development. Present in every stage are important emotional tasks associated with career issues (e.g., moving from fantasy to realistic options; anxiety that comes with decision making or implementation of career decisions; challenge to advance and succeed). Future research could examine the use of cognitive reappraisal techniques in career counseling with individuals facing specific emotional tasks in the various stages of career development (e.g., exploration, establishment, maintenance). There are specific tasks at each developmental stage that may require change in the individual's meaning system. For example, in the exploration stage, researchers could test the effects on emotional regulation systems of different reappraisal techniques in helping the client move from fantasy to realistic career options (e.g., even though it is sad that I cannot be an astronaut, there are plenty of professional opportunities to explore the universe). In the establishment stage, researchers could do similar testing using cognitive reappraisal helping clients stabilize in their careers (e.g., even though I'm missing the freedom and the adventure, it is good to feel safe and secure and know where I'm going).

Contrasting with the top-down emotional regulation and cognitive reappraisal, clinicians have recently been exploring the use of mindfulness as a way of regulating emotional and cognitive experience. Tang (2011) introduced a mindfulness variation referred to as integrative body-mind training (IBMT). IBMT is a type of meditation integrating relaxation, imagery, and mindfulness. This brief intervention was found not only to reduce stress and improve attention but also to have a substantial impact on the brain at the functional and structural level. Particularly relevant is the finding on the impact of IBMT intervention on the increase of the level of fractional anisotropy (an index of white matter integrity) of white matter connections with the anterior cingulate region (i.e., anterior corona radiate). Similarly to what was found in the studies of cognitive reappraisal, different brain regions were found to be active in different states of IBMT training. During the initial stages of training, an effort of cognitive control is requiring the involvement of the attention network that seems to be evident (i.e., lateral prefrontal cortex and parietal cortex). However, as the individual masters the technique, the control seems to shift the anterior cingulate, the insula, and the striatum, involving less effort of attention control and maintenance of a better body-mind integration (insula) (Tang et al., 2012).

We suggest that counseling psychologists engage in interdisciplinary research with neuroscientists to investigate the mechanisms responsible for the impact of discrete counseling interventions (e.g., cognitive reframing; IBMT) in psychological processes associated with the different brain network systems. Counseling psychology researchers could also use findings from neuroscience to refine our practice and test the effectiveness of modified counseling interventions targeting emotional regulation. Warren et al. (2010) found that individuals with insecure attachment regulate their emotions by mobilizing increased neurocognitive resources of top-down cognitive control (involving the left dorsolateral prefrontal cortex, anterior cingulate cortex, and superior frontal gyrus). Given the demonstrated effects of IBMT on decreasing the attention control by shifting activations toward the anterior cingulate, future researchers could use pretest, posttest methods to evaluate the effectiveness of IBMT in reducing anxiety for indi- 
viduals with secure versus insecure attachment in order to determine which groups could benefit most from this type of intervention. If differences exist, researchers could assess whether psychotherapy can attenuate the effects of these factors and, through practice, allow all individuals to benefit from these strategies in a similar manner (e.g., based on the finding that less effort at attention control was needed after and individuals achieved mastery of the technique, per Tang et al., 2012).

\section{Integrating Neuroscience Into Contextual Counseling Interventions}

Neuroscience researchers have identified several psychosocial mechanisms that promote neuroplasticity by impacting neurogenesis and synaptogenesis in different neural systems. Four of those mechanisms that we believe warrant investigation by counseling psychology researchers are nurturing and caring, healthy behavior, and environmental enrichment. Animal research has consistently shown that nurturing and caring behaviors have a strong, longlasting effect on the neurodevelopment of the offspring. For example, research on maternal behavior among rats indicated pups from mothers showing high levels of licking and grooming during the first week after birth show less vulnerability to stress, with a decreased corticosterone response (Bagot et al., 2012). Similar research is now being conducted with humans, showing that caring and nurturing attitudes have a significant impact in children's brain development. For example, Milgrom et al. (2010), trained pregnant women in the use of specific strategies to reduce stressful experiences and influence infants' brain development. The training of mothers was focused on promoting an overall caring and nurturing attitude (e.g., facial expressions, movement and massage, skin contact, and multisensory stimulation). Results from this study showed that white matter connectivity and maturation was significantly impacted in the trained group, which suggests that this type of parenting training could positively influence neurodevelopment of children. Future researchers could compare the effectiveness of different components of parenting training in order to isolate the active mechanisms responsible for the induction of brain plasticity. For example, researchers could compare two identical parenting interventions but add multisensory stimulation to one of interventions to assess the role of that component on positive neurodevelopment of children as well as factors known to be influenced by nurturing caregivers, such as attachment security (Bowlby, 1973) and subjective well-being (Diener et al., 1985).

The practice of healthy behaviors, particularly those associated with nutrition and exercise, have also been linked to positive brain plasticity. For example, Griffin et al. (2011) found that short periods of intense physical exercise increased the levels of brainderived neurotrophic factor (BDNF) and improved performance in a memory task (i.e., face-name matching). Similarly, healthy dietary habits have been found to contribute to cognitive enhancement of the mechanisms of positive neuroplasticity. Witte, Fobker, Gellner, Knecht, and Flöel (2009) found that a $30 \%$ caloric restricted diet (when compared with a $20 \%$ increase in unsaturated fatty acids and control) had significant effects in terms of memory as well as in biological markers underlying processes of synaptic plasticity such as improved insulin sensitivity and reduced inflammatory activity. Finally, environmental enrichment is one of the most widely proven contextual mechanisms responsible for pro- moting positive neuroplasticity. Most of the research done so far has used animal models. This research has shown strong effects of environmental enrichment on brain networks associated with sensory and cognitive processes (Baroncelli et al., 2010). In humans, environmental enrichment is most often done by increasing in patterns of cognitive, social, and physical activity. Miller, Colella, and Green (2013) looked at the effects of postmorbid environmental enrichment on hippocampal atrophy in traumatic brain injury and found that environmental enrichment activities were protective of hippocampal atrophy in these participants.

Building on some of the findings discussed above, counseling psychology researchers should seek to examine the natural and intentional manipulation of these contextual factors, such as parental caring and nurturing attitudes, the promotion of healthy behaviors, and environmental enrichment, as a way of enhancing neurodevelopment across developmental domains. Future researchers could examine, for example, (a) the role of attachment in modulating the emotion regulation network and influencing tasks across developmental domains (cognitive, emotional, vocational, social, and identity development), (b) the mechanisms by which aerobic exercise promotes cognitive development and enhances attention networks, and (c) the influence of exposure to different patterns of culture diversity on social cognitive networks and the correlative identity developmental tasks.

\section{Concluding Remarks}

It is our hope that this article will serve as a call to action for counseling psychologists to pursue research that integrates neuroscience with traditional counseling psychology paradigms. We argue for a transdiagnostic developmental perspective for understanding our clients and have provided directions for integrating neuroscience into future process and outcome research as well as future research directions regarding the effects of contextual counseling interventions. We suggest future research is needed to explore the interaction of interpersonal and environmental factors, psychological processes, brain networks, and development, including cognitive, social, emotional, identity, and vocational development.

\section{References}

Bagot, R. C., Zhang, T.-Y., Wen, X., Nguyen, T. T. T., Nguyen, H.-B., Diorio, J., . . Meaney, M. J. (2012). Variations in postnatal maternal care and the epigenetic regulation of metabotropic glutamate receptor 1 expression and hippocampal function in the rat. Proceedings of the National Academy of Sciences, USA, 109(Suppl. 2), 17200-17207. doi:10.1073/pnas.1204599109

Baroncelli, L., Braschi, C., Spolidoro, M., Begenisic, T., Sale, A., \& Maffei, L. (2010). Nurturing brain plasticity: Impact of environmental enrichment. Cell Death and Differentiation, 17, 1092-1093.

Barsaglini, A., Sartori, G., Benetti, S., Pettersson-Yeo, W., \& Mechelli, A (in press). The effects of psychotherapy on brain function: A systematic and critical review. Progresses in Neurobiology.

Bowlby, J. (1973). Attachment and loss: Vol. 2. Separation: Anxiety and anger. New York, NY: Basic Books.

Buboltz, W., Deemer, E., \& Hoffman, R. (2010). Content analysis of the Journal of Counseling Psychology: Buboltz, Miller, and Williams (1999) 11 years later. Journal of Counseling Psychology, 57, 368-375.

Buckholtz, J. W., \& Meyer-Lindenberg, A. (2012). Psychopathology and the human connectome: Toward a transdiagnostic model of risk for 
mental illness. Neuron, 74, 990-1004. doi:10.1016/j.neuron.2012.06 .002

Buhle, J. T., Silvers, J. A., Wager, T. D., Lopez, R., Onyemekwu, C., Kober, H., . . . Ochsner, K. N. (in press). Cognitive reappraisal of emotion: A meta-analysis of human neuroimaging studies. Cerebral Cortex.

Cacioppo, J., Berntson, G., \& Nusbaum, H. (2008). Neuroimaging as a new tool in the toolbox of psychological science. Current Directions in Psychological Science, 17, 62-67. doi:10.1111/j.1467-8721.2008 .00550.x

Cowan, W. M., Harter, D. H., \& Kandel, E. R. (2000). The emergence of modern neuroscience: Some implications for neurology and psychiatry. Annual Review of Neuroscience, 23, 343-391.

Diener, E., Emmons, R. A., Larson, R. J., \& Griffin, S. (1985). The Satisfaction With Life Scale. Journal of Personality Assessment, 49, 71-75. doi:10.1207/s15327752jpa4901_13

Goldapple, K., Segal, Z., Garson, C., Lau, M., Bieling, P., Kennedy, S., \& Mayberg, H. (2004). Modulation of cortical-limbic pathways in major depression: Treatment-specific effects of cognitive behavior therapy. Archives of General Psychiatry, 61, 34-41. doi:10.1001/archpsyc.61 .1 .34

Hill, C. E., Helms, J. E., Tichenor, V., Spiegel, S. B., O'Grady, K. E., \& Perry, E. S. (1988). Effects of therapist response modes in brief psychotherapy. Journal of Counseling Psychology, 35, 222-233. doi:10.1037/ 0022-0167.35.3.222

Hong, S.-B., Zalesky, A., Cocchi, L., Fornito, A., Choi, E.-J., Kim, H.-H., . . . Yi, S.-H. (2013). Decreased functional brain connectivity in adolescents with Internet addiction. PLOS ONE, 8, e57831. doi:10.1371/ journal.pone. 0057831

Ivey, A., Ivey, M., \& Zalaquett, C. (2013). Intentional interviewing and counseling: Facilitating client development in a multicultural world (7th ed.), Belmont, CA: Brooks/Cole/Cengage.

Ivey, A., \& Zalaquett, C. J. (2011). Neuroscience and counseling: Central issue for social justice. Journal for Social Action in Counseling and Psychology, 3, 103-116.

Kandel, E. (1998). A new intellectual framework for psychiatry. American Journal of Psychiatry, 155, 457-469.

Kandel, E. (2000). The molecular biology of memory storage: A dialog between genes and synapses. Bioscience Reports, 2, 545-565.

Katehakis, A. (2009). Affective neuroscience and the treatment of sexual addiction. Sexual Addiction \& Compulsivity, 16, 1-31. doi:10.1080/ 10720160802708966

Kazdin, A. E. (2007). Mediators and mechanisms of change in psychotherapy research. Annual Review of Clinical Psychology, 3, 1-27. doi: 10.1146/annurev.clinpsy.3.022806.091432

Kumari, V. (2006). Do psychotherapies produce neurobiological effects? Acta Neuropsychiatrica, 18, 61-70. doi:10.1111/j.1601-5215.2006 .00127.x

Leite, J., Carvalho, S., Fregni, F., Boggio, P. S., \& Gonçalves, O. F. (2013). The effects of cross-hemispheric dorsolateral prefrontal cortex transcranial direct current stimulation (tDCS) on task switching. Brain Stimulation, 6, 660-667. doi:10.1016/j.brs.2012.10.006

Leskin, L. P., \& White, P. M. (2007). Attentional networks reveal executive function deficits in posttraumatic stress disorder. Neuropsychology, 21, 275-284. doi:10.1037/0894-4105.21.3.275

McRae, K., Ciesielski, B., \& Gross, J. J. (2012). Unpacking cognitive reappraisal: Goals, tactics, and outcomes. Emotion, 12, 250-255.

Milgrom, J., Newnham, C., Anderson, P. J., Doyle, L. W., Gemmill, A. W., Lee, K., . . Inder, T. (2010). Early sensitivity training for parents of preterm infants: Impact on the developing brain. Pediatric Research, 67, 330-335.

Miller, L. S., Colella, B., \& Green, R. E. (2013). Environmental enrichment may protect against hippocampal atrophy in the chronic stages of traumatic brain injury. Frontiers in Human Neuroscience, 7, 506-507. doi:10.3389/fnhum.2013.00506

Oliveira-Silva, P., \& Gonçalves, Ó. F. (2011). Responding empathically: A question of heart, not a question of skin. Applied Psychophysiology and Biofeedback, 36, 201-207. doi:10.1007/s10484-011-9161-2

Penadés, R., Pujol, N., Catalán, R., Massana, G., Rametti, G., García-Rizo, C., . . Junqué, C., (2013). Brain effects of cognitive remediation therapy in schizophrenia: A structural and functional neuroimaging study. Biological Psychiatry, 73, 1015-1023.

Pinheiro, A. P., del Re, E., Nestor, P., McCarley, R. W., Gonçalves, O. F., \& Niznikiewicz, M. (2013). Interactions between mood and the structure of semantic memory: Event-related potentials evidence. Social Cognitive \& Affective Neuroscience, 8, 579-594. doi:10.1093/scan/nss035

Rochman, D., \& Diamond, G. M. (2008). From unresolved anger to sadness: Identifying physiological correlates. Journal of Counseling Psychology, 55, 96-105. doi:10.1037/0022-0167.55.1.96

Sampaio, A., Soares, J., Coutinho, J., Sousa, N., \& Gonçalves, O. F. (in press). The Big Five default brain: Functional evidence. Brain Structure and Function.

Sescousse, G., \& den Ouden, H. M. (2013). Gambling rats and gambling addiction: Reconciling the role of dopamine in irrationality. The Journal of Neuroscience, 33, 3256-3258. doi:10.1523/JNEUROSCI.5828-12 .2013

Silvers, J. A., Buhle, J. T., \& Ochsner, K. N. (2013). The neuroscience of emotion regulation: Basic mechanisms and their role in development, aging and psychopathology. In K. N. Ochsner \& S. M. Kosslyn (Eds.), The handbook of cognitive neuroscience (Vol. I, pp. 1-40). New York, NY: Oxford University Press.

Super, D. W. (1980). A life-span, life-space approach to career development. Journal of Vocational Behavior, 16, 282-298. doi:10.1016/00018791(80)90056-1

Tang, Y. Y. (2011). Mechanisms of integrative body-mind training. Neuroscience Bulletin, 27, 383-388. doi:10.1007/s12264-011-1141-2

Tang, Y. Y., Rothbart, M. K., \& Posner, M. I. (2012). Neural correlates of establishing, maintaining, and switching brain states. Trends in Cognitive Sciences, 16, 330-337. doi:10.1016/j.tics.2012.05.001

Tottenham, N., Hare, T. A., Quinn, B. T., McCarry, T. W., Nurse, M., Gilhooly, T., . . Casey, B. J. (2010). Prolonged institutional rearing is associated with atypically large amygdala volume and difficulties in emotion regulation. Developmental Science, 13, 46-61. doi:10.1111/j .1467-7687.2009.00852.x

Valenstein, E. (1998). Blaming the brain: The truth about drugs and mental health. New York, NY: Free Press.

Warren, S. L., Bost, K. K., Roisman, G. I., Silton, R. L., Spielberg, J. M., Engels, A. S., . . Heller, W. (2010). Effects of adult attachment and emotional distractors on brain mechanisms of cognitive control. Psychological Science, 21, 1818-1826. doi:10.1177/0956797610388809

Witte, A. V., Fobker, M., Gellner, R., Knecht, S., \& Flöel, A. (2009). Caloric restriction improves memory in elderly humans. Proceedings of the National Academy of Sciences, USA, 106, 1255-1260. doi:10.1073/ pnas.0808587106

Received November 13, 2013

Revision received January 25, 2014 Accepted March 17, 2014 